\title{
A sum of negative degrees of the gaps values in 2 and 3-generated numerical semigroups
}

\author{
Leonid G. Fel ${ }^{a *}$, Takao Komatsu ${ }^{b}$, \\ Ade Irma Suriajaya ${ }^{c}$ \\ ${ }^{a}$ Department of Civil Engineering, Technion, \\ Haifa 32000, Israel \\ lfel@technion.ac.il \\ ${ }^{b}$ Department of Mathematical Sciences, School of Science, \\ Zhejiang Sci-Tech University, \\ Hangzhou 310018, China \\ komatsu@zstu.edu.cn \\ ${ }^{c}$ Faculty of Mathematics, Kyushu University, \\ 744 Motooka, Nishi-ku, \\ Fukuoka 819-0395, Japan \\ adeirmasuriajaya@math.kyushu-u.ac.jp \\ Submitted: January 25, 2020 \\ Accepted: August 27, 2020 \\ Published online: September 2, 2020
}

\begin{abstract}
We show explicit expressions for an inverse power series over the gaps values of numerical semigroups generated by two and three integers. As an application, a set of identities of the Hurwitz zeta functions is derived.

Keywords: numerical semigroups, gaps and non-gaps, the Hurwitz zeta function

$M S C$ : Primary 20M14; Secondary 11P81
\end{abstract}

\footnotetext{
*The research of LGF was supported in part by the Kamea Fellowship.
} 


\section{Introduction}

A sum of integer powers of gaps values in numerical semigroups $S_{m}=\left\langle d_{1}, \ldots, d_{m}\right\rangle$ with $\operatorname{gcd}\left(d_{1}, \ldots, d_{m}\right)=1$, is referred often as the semigroup series

$$
g_{n}\left(S_{m}\right)=\sum_{s \in \mathbb{N} \backslash S_{m}} s^{n}, \quad n \in \mathbb{Z},
$$

where $\mathbb{N} \backslash S_{m}$ is known as the set of gaps of $S_{m}$ and $g_{0}\left(S_{m}\right)$ is called the genus of $S_{m}$. The semigroup series $g_{n}\left(S_{m}\right)$ has been attractive by many researchers for $n \geq 0$. In particular, an explicit expression of $g_{n}\left(S_{2}\right)$ and implicit expression of $g_{n}\left(S_{3}\right)$ were given in [6] and [4], respectively. However, the series $g_{n}\left(S_{m}\right)$ for negative integers $n$ has not seemingly treated so often. In this paper we derive a formula for semigroup series $g_{-n}\left(S_{2}\right)=\sum_{s \in \mathbb{N} \backslash S_{2}} s^{-n}$ and $g_{-n}\left(S_{3}\right)=\sum_{s \in \mathbb{N} \backslash S_{3}} s^{-n}(n \geq 1)$. In fact, it will be known that such series are related with zeta functions in Number theory.

Consider a numerical semigroup $S_{2}=\left\langle d_{1}, d_{2}\right\rangle$, generated by two integers $d_{1}, d_{2} \geq$ 2 with $\operatorname{gcd}\left(d_{1}, d_{2}\right)=1$. Here, the Hilbert series $H\left(z ; S_{2}\right)$ and the gaps generating function $\Phi\left(z ; S_{2}\right)$ are given as

$$
H\left(z ; S_{2}\right)=\sum_{s \in S_{2}} z^{s} \text { and } \Phi\left(z ; S_{2}\right)=\sum_{s \in \mathbb{N} \backslash S_{2}} z^{s},
$$

respectively, satisfying

$$
H\left(z ; S_{2}\right)+\Phi\left(z ; S_{2}\right)=\frac{1}{1-z} \quad(z<1)
$$

where $\min \left\{\mathbb{N} \backslash S_{2}\right\}=1$, and $\max \left\{\mathbb{N} \backslash S_{2}\right\}=d_{1} d_{2}-d_{1}-d_{2}$ is called the Frobenius number and is denoted by $F_{2}$. A rational representation (Rep) of $H\left(z ; S_{2}\right)$ is given by

$$
H\left(z ; S_{2}\right)=\frac{1-z^{d_{1} d_{2}}}{\left(1-z^{d_{1}}\right)\left(1-z^{d_{2}}\right)} .
$$

We introduce a new generating function $\Psi_{1}\left(z ; S_{2}\right)$, defined by

$$
\Psi_{1}\left(z ; S_{2}\right)=\int_{0}^{z} \frac{\Phi\left(t ; S_{2}\right)}{t} \mathrm{~d} t=\sum_{s \in \mathbb{N} \backslash S_{2}} \frac{z^{s}}{s} \quad \text { with } \quad \Psi_{1}\left(1 ; S_{2}\right)=g_{-1}\left(S_{2}\right) .
$$

Substituting (1.1) into (1.3), we obtain

$$
\Psi_{1}\left(z ; S_{2}\right)=\int_{0}^{z}\left(\frac{1}{1-t}-H\left(t ; S_{2}\right)\right) \frac{\mathrm{d} t}{t} .
$$

Since $\left(1-t^{d_{i}}\right)^{-1}=\sum_{k_{i}=0}^{\infty} t^{k_{i} d_{i}}$, by substituting (1.4) into (1.2), we obtain

$$
H\left(t ; S_{2}\right)=\sum_{k_{1}, k_{2}=0}^{\infty} t^{k_{1} d_{1}+k_{2} d_{2}}-\sum_{k_{1}, k_{2}=0}^{\infty} t^{k_{1} d_{1}+k_{2} d_{2}+d_{1} d_{2}} .
$$


Indeed, an expression (1.5) is an infinite series with degrees $s=k_{1} d_{1}+k_{2} d_{2}$ running over all nodes in the following sublattice $\mathbb{K}$ of the integer lattice $\mathbb{Z}_{2}$.

$$
\mathbb{K}=\{0,0\} \cup \mathbb{K}_{1} \cup \mathbb{K}_{2}, \quad\left\{\begin{array}{l}
\mathbb{K}_{1}=\left\{1 \leq k_{1} \leq d_{2}-1, \quad k_{2}=0\right\}, \\
\mathbb{K}_{2}=\left\{0 \leq k_{1} \leq d_{2}-1, \quad 1 \leq k_{2} \leq \infty\right\} .
\end{array}\right.
$$

In Figure 1, as an example, we present a part of the integer lattice $\mathbb{K}$ for the numerical semigroup

$$
\langle 5,8\rangle=\{0,5,8,10,13,15,16,18,20,21,23,24,25,26,28, \longmapsto\},
$$

where the symbol $\longmapsto$ denotes an infinite set of positive integers exceeding 28 .

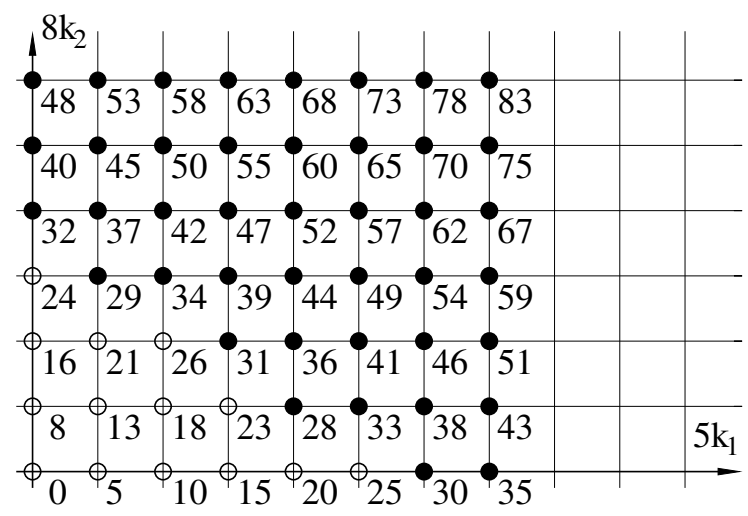

Figure 1: A part of the integer lattice $\mathbb{K} \subset \mathbb{Z}_{2}$ for the numerical semigroup $\langle 5,8\rangle$. The nodes mark the non-gaps of semigroup: the values, assigned to the black and white nodes, exceed and precede $F_{2}=27$, respectively.

Proposition 1.1. There exists a bijection between the infinite set of nodes in the integer lattice $\mathbb{K}$ and an infinite set of non-gaps of the semigroup $\left\langle d_{1}, d_{2}\right\rangle$.

Proof. We have to prove two statements of existence and uniqueness:

1) Every $s \in\left\langle d_{1}, d_{2}\right\rangle$ has its Rep node in $\mathbb{K}$,

2) All $s \in\left\langle d_{1}, d_{2}\right\rangle$ have their Rep nodes in $\mathbb{K}$ only once.

1) Let $s \in\left\langle d_{1}, d_{2}\right\rangle$ be given. Then by definition of $\left\langle d_{1}, d_{2}\right\rangle$ an integer $s$ has Rep,

$$
s=k_{1} d_{1}+k_{2} d_{2}, \quad k_{1}, k_{2} \in \mathbb{Z}, \quad k_{1}, k_{2} \geq 0 .
$$

Choose $s$ such that $k_{1}=p d_{2}+q$, where $p=\left\lfloor k_{1} / d_{2}\right\rfloor$, namely, $0 \leq q \leq d_{2}-1$, and $\lfloor x\rfloor$ denotes the integer part of a real number $x$. Then Rep (1.7) is expressed as

$$
s=q d_{1}+\left(k_{2}+p d_{1}\right) d_{2},
$$


and $s$ has its Rep node in $\mathbb{K}$.

2) By way of contradiction, assume that there exist two nodes $\left\{k_{1}, k_{2}\right\} \in \mathbb{K}$ and $\left\{l_{1}, l_{2}\right\} \in \mathbb{K}$ such that

$$
\begin{aligned}
& k_{1} d_{1}+k_{2} d_{2}=l_{1} d_{1}+l_{2} d_{2}, \\
& 0 \leq k_{1}, l_{1} \leq d_{2}-1, \quad 0 \leq k_{2}, l_{2} \leq \infty, \quad k_{1}>l_{1}, \quad k_{2}<l_{2}
\end{aligned}
$$

namely, that there exists such $s \in\left\langle d_{1}, d_{2}\right\rangle$ which has two different Rep nodes in $\mathbb{K}$. Rewrite equality (1.8) as follows.

$$
\left(k_{1}-l_{1}\right) d_{1}=\left(l_{2}-k_{2}\right) d_{2} .
$$

Since $\operatorname{gcd}\left(d_{1}, d_{2}\right)=1$, the equality (1.9) implies that

$$
k_{1}-l_{1}=b d_{2} \quad(b \geq 1) \quad \Longrightarrow \quad k_{1}=l_{1}+b d_{2} \quad \Longrightarrow \quad k_{1} \geq d_{2},
$$

contradicting the assumption $\left\{k_{1}, k_{2}\right\} \in \mathbb{K}$.

\section{A sum of the inverse gaps values $g_{-1}\left(S_{2}\right)$}

Rewrite the integral in (1.4) as follows.

$$
\Psi_{1}\left(z ; S_{2}\right)=\int_{0}^{z}\left(\sum_{k=0}^{\infty} t^{k-1}-\frac{H\left(t ; S_{2}\right)}{t}\right) \mathrm{d} t
$$

where

$$
\begin{aligned}
\frac{H\left(t ; S_{2}\right)}{t} & =\sum_{j=0}^{2} h_{j}\left(t ; S_{2}\right), & h_{0}\left(t ; S_{2}\right) & =\frac{1}{t} \\
h_{1}\left(t ; S_{2}\right) & =\sum_{k_{1}=1}^{d_{2}-1} t^{k_{1} d_{1}-1}, & h_{2}\left(t ; S_{2}\right) & =\sum_{k_{1}, k_{2} \in \mathbb{K}_{2}} t^{k_{1} d_{1}+k_{2} d_{2}-1} .
\end{aligned}
$$

By integration we obtain from (2.1),

$$
\Psi_{1}\left(z ; S_{2}\right)=\sum_{k=1}^{\infty} \frac{z^{k}}{k}-\frac{1}{d_{1}} \sum_{k_{1}=1}^{d_{2}-1} \frac{z^{k_{1} d_{1}}}{k_{1}}-\sum_{k_{1}, k_{2} \in \mathbb{K}_{2}} \frac{z^{k_{1} d_{1}+k_{2} d_{2}}}{k_{1} d_{1}+k_{2} d_{2}},
$$

and deduce by (1.3) and (1.6),

$$
g_{-1}\left(S_{2}\right)=\sum_{k=1}^{\infty} \frac{1}{k}-\sum_{k_{1}, k_{2} \in \mathbb{K}_{2}} \frac{1}{k_{1} d_{1}+k_{2} d_{2}}-\frac{1}{d_{1}} \sum_{k_{1}=1}^{d_{2}-1} \frac{1}{k_{1}} .
$$


By Proposition 1.1, after subtraction in (2.3) there is a finite number of terms left, since all terms, which exceed $F_{2}$ in the two first infinite series in (2.3), are cancelled. To emphasize that fact, we represent formula (2.3) as follows.

$$
g_{-1}\left(S_{2}\right)=\sum_{k=1}^{c_{2}} \frac{1}{k}-\sum_{k_{1}, k_{2} \in \mathbb{K}_{2}}^{k_{1} d_{1}+k_{2} d_{2} \leq c_{2}} \frac{1}{k_{1} d_{1}+k_{2} d_{2}}-\frac{1}{d_{1}} \sum_{k_{1}=1}^{d_{2}-1} \frac{1}{k_{1}},
$$

where $c_{2}=F_{2}+1$ is called the conductor of semigroup $S_{2}$.

\section{A sum of the negative degrees of gaps values $g_{-n}\left(S_{2}\right)$}

We generalize formula $(2.2)$ and introduce a new generating function $\Psi_{n}\left(z ; S_{2}\right)$ $(n \geq 2)$

$$
\Psi_{n}\left(z ; S_{2}\right)=\int_{0}^{z} \frac{\mathrm{d} t_{1}}{t_{1}} \int_{0}^{t_{1}} \frac{\mathrm{d} t_{2}}{t_{2}} \ldots \int_{0}^{t_{n-1}} \Phi\left(t_{n} ; S_{2}\right) \frac{\mathrm{d} t_{n}}{t_{n}}=\sum_{s \in \mathbb{N} \backslash S_{2}} \frac{z^{s}}{s^{n}},
$$

where $\Psi_{n}\left(1 ; S_{2}\right)=g_{-n}\left(S_{2}\right)$ and satisfies the following recursive relation.

$$
\begin{aligned}
& \Psi_{k+1}\left(t_{n-k-1} ; S_{2}\right)=\int_{0}^{t_{n-k-1}} \frac{\mathrm{d} t_{n-k}}{t_{n-k}} \Psi_{k}\left(t_{n-k} ; S_{2}\right), \quad k \geq 0, \\
& \Psi_{0}\left(t_{n} ; S_{2}\right)=\Phi\left(t_{n-1} ; S_{2}\right), \quad t_{0}=z .
\end{aligned}
$$

Namely,

$$
\begin{aligned}
& \Psi_{1}\left(t_{n-1} ; S_{2}\right)=\int_{0}^{t_{n-1}} \frac{\mathrm{d} t_{n}}{t_{n}} \Psi_{0}\left(t_{n} ; S_{2}\right), \\
& \Psi_{2}\left(t_{n-2} ; S_{2}\right)=\int_{0}^{t_{n-2}} \frac{\mathrm{d} t_{n-1}}{t_{n-1}} \Psi_{1}\left(t_{n-1} ; S_{2}\right), \ldots .
\end{aligned}
$$

By integration in (3.1), we obtain

$$
\Psi_{n}\left(z ; S_{2}\right)=\sum_{k=1}^{\infty} \frac{z^{k}}{k^{n}}-\frac{1}{d_{1}^{n}} \sum_{k_{1}=1}^{d_{2}-1} \frac{z^{k_{1} d_{1}}}{k_{1}^{n}}-\sum_{k_{1}, k_{2} \in \mathbb{K}_{2}} \frac{z^{k_{1} d_{1}+k_{2} d_{2}}}{\left(k_{1} d_{1}+k_{2} d_{2}\right)^{n}} .
$$

Thus, for $z=1$ we have

$$
g_{-n}\left(S_{2}\right)=\sum_{k=1}^{\infty} \frac{1}{k^{n}}-\sum_{k_{1}=0}^{d_{2}-1} \sum_{k_{2}=1}^{\infty} \frac{1}{\left(k_{1} d_{1}+k_{2} d_{2}\right)^{n}}-\frac{1}{d_{1}^{n}} \sum_{k_{1}=1}^{d_{2}-1} \frac{1}{k_{1}^{n}}, \quad n \geq 2 .
$$


Denoting the ratio $d_{1} / d_{2}$ by $\delta$, we can rewrite $(3.2)$ as

$$
g_{-n}\left(S_{2}\right)=\sum_{k=1}^{\infty} \frac{1}{k^{n}}-\frac{1}{d_{2}^{n}} \sum_{k_{2}=1}^{\infty} \frac{1}{k_{2}^{n}}-\frac{1}{d_{2}^{n}} \sum_{k_{1}=1}^{d_{2}-1} \sum_{k_{2}=1}^{\infty} \frac{1}{\left(k_{1} \delta+k_{2}\right)^{n}}-\frac{1}{d_{1}^{n}} \sum_{k_{1}=1}^{d_{2}-1} \frac{1}{k_{1}^{n}} .
$$

Making use of the Hurwitz $\zeta(n, q)=\sum_{k=0}^{\infty}(k+q)^{-n}$ and Riemann zeta functions $\zeta(n)=\zeta(n, 1)$, we represent the last formula as follows.

$$
g_{-n}\left(S_{2}\right)=\left(1-\frac{1}{d_{2}^{n}}\right) \zeta(n)-\frac{1}{d_{2}^{n}} \sum_{k_{1}=1}^{d_{2}-1} \zeta\left(n, k_{1} \delta\right), \quad n \geq 2 .
$$

On interchanging the generators $d_{1}$ and $d_{2}$ in (3.3), we obtain an alternative expression for $g_{-n}\left(S_{2}\right)$ :

$$
g_{-n}\left(S_{2}\right)=\left(1-\frac{1}{d_{1}^{n}}\right) \zeta(n)-\frac{1}{d_{1}^{n}} \sum_{k_{2}=1}^{d_{1}-1} \zeta\left(n, \frac{k_{2}}{\delta}\right) .
$$

\section{Symmetric 3-generated numerical semigroup}

We deal with symmetric numerical semigroup $S_{3}=\left\langle d_{1}, d_{2}, d_{3}\right\rangle$ generated by three integers with the Hilbert series $H\left(z ; S_{3}\right)$, satisfying minimal relations,

$$
H\left(z ; S_{3}\right)=\frac{\left(1-z^{a_{22} d_{2}}\right)\left(1-z^{a_{33} d_{3}}\right)}{\left(1-z^{d_{1}}\right)\left(1-z^{d_{2}}\right)\left(1-z^{d_{3}}\right)} \quad\left(a_{22}, a_{33} \geq 2\right),
$$

with $a_{11} d_{1}=a_{22} d_{2}, a_{33} d_{3}=a_{31} d_{1}+a_{32} d_{2}$ (see [3]). In this section, we prove a statement which is necessary to establish the convergence for $g_{1}\left(z, S_{3}\right)$, namely, the difference between two divergent infinite series is convergent

$$
g_{1}\left(z, S_{3}\right)=\sum_{k=1}^{\infty} \frac{1}{k}-\sum_{k_{2}=0}^{a_{22}-1} \sum_{k_{3}=0}^{a_{33}-1} \sum_{k_{1}=0}^{\infty} \frac{1}{k_{1} d_{1}+k_{2} d_{2}+k_{3} d_{3}}, \quad \sum_{j=1}^{3} k_{j} \geq 1 .
$$

The idea is to prove that after cancellation of identical terms, a finite number of terms is left in (4.2).

We consider the sublattice $\widetilde{\mathbb{L}}=\mathbb{L} \cup\{0,0,0\}$ of the integer lattice $\mathbb{Z}_{3}$, where

$$
\mathbb{L}=\bigcup_{\substack{k_{1}=0 \\ k_{1}+k_{2}+k_{3} \geq 1}}^{\infty} \mathbb{L}_{k_{1}}, \quad \mathbb{L}_{k_{1}}=\bigcup_{k_{2}, k_{3}}^{\infty}\left\{k_{1}, k_{2}, k_{3}\right\}
$$

with $0 \leqq k_{2}<a_{22}$ and $0 \leq k_{3}<a_{33}$. In Figure 2, we present a part of the integer lattice $\widetilde{\widetilde{\mathbb{L}}}$ for the numerical semigroup $\langle 4,7,10\rangle$.

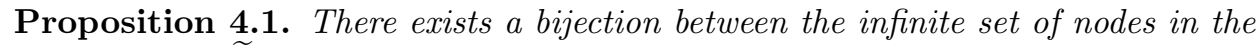
integer lattice $\widetilde{\mathbb{L}}$ and an infinite set of non-gaps of the semigroup $\left\langle d_{1}, d_{2}, d_{3}\right\rangle$. 


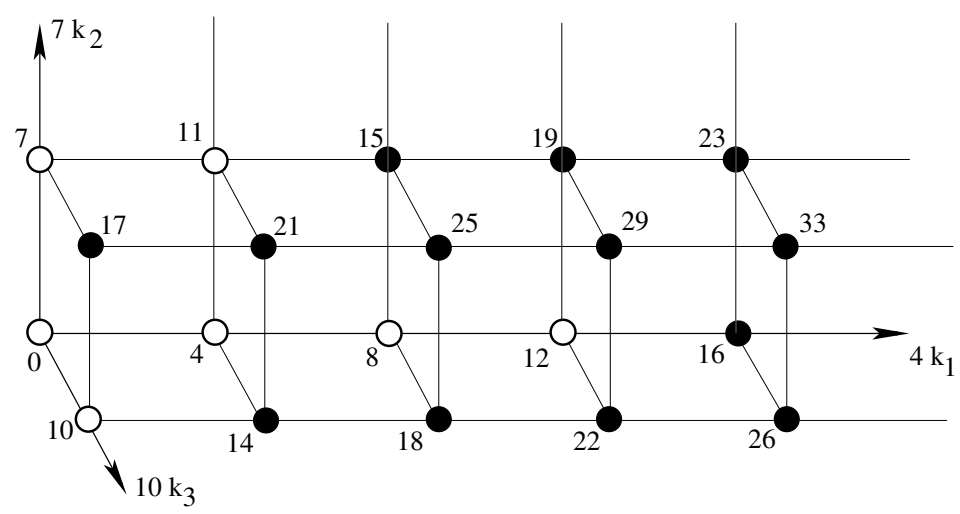

Figure 2: A part of the integer lattice $\widetilde{\mathbb{L}} \subset \mathbb{Z}_{3}$ for $\langle 4,7,10\rangle$. The nodes mark the non-gaps of semigroup: the values, assigned to the black and white nodes, exceed and precede the Frobenius number $F_{3}=13$.

Proof. We have to prove both existence and uniqueness.

1) Every $s \in\left\langle d_{1}, d_{2}, d_{3}\right\rangle$ has its representative node in $\widetilde{\mathbb{L}}$.

2) All $s \in\left\langle d_{1}, d_{2}, d_{3}\right\rangle$ have their representative nodes in $\widetilde{\mathbb{L}}$ only once.

1) Let $s \in\left\langle d_{1}, d_{2}, d_{3}\right\rangle$ be given. Then by definition of $\left\langle d_{1}, d_{2}, d_{3}\right\rangle$ an integer $s$ has a representation,

$$
s=k_{1} d_{1}+k_{2} d_{2}+k_{3} d_{3}, \quad 0 \leq k_{1}, k_{2}, k_{3}<\infty .
$$

Choose $s$ such that

$$
k_{2}=p_{2} a_{22}+q_{2}, \quad k_{3}=p_{3} a_{33}+q_{3}, \quad \text { namely, } \quad p_{2}=\left\lfloor\frac{k_{2}}{a_{22}}\right\rfloor, p_{3}=\left\lfloor\frac{k_{3}}{a_{33}}\right\rfloor \text {, }
$$

$p_{2}, p_{3}, q_{2}, q_{3} \in \mathbb{Z}, \quad p_{2}, p_{3} \geq 0, \quad 0 \leq q_{2}<a_{22}, \quad 0 \leq q_{3}<a_{33}$.

By substituting (4.4) into (4.3), we get

$$
s=k_{1} d_{1}+\left(p_{2} a_{22}+q_{2}\right) d_{2}+\left(p_{3} a_{33}+q_{3}\right) d_{3} .
$$

Combining (4.5) with minimal relations (4.1), we obtain

$$
\begin{aligned}
s & =\left(k_{1}+p_{2} a_{11}\right) d_{1}+p_{3}\left(a_{31} d_{1}+a_{32} d_{2}\right)+q_{2} d_{2}+q_{3} d_{3} \\
& =\left(k_{1}+p_{2} a_{11}+p_{3} a_{31}\right) d_{1}+\left(p_{3} a_{32}+q_{2}\right) d_{2}+q_{3} d_{3} .
\end{aligned}
$$

If $p_{3} a_{32}+q_{2}<a_{22}$, then $s$ has its representative node in $\widetilde{\mathbb{L}}$. But, if $p_{3} a_{32}+q_{2} \geq a_{22}$, let us write

$$
p_{3} a_{32}+q_{2}=p_{4} a_{22}+q_{4}, \quad p_{4} \geq 0, \quad 0 \leq q_{4}<a_{22}, \quad p_{4}=\left\lfloor\frac{p_{3} a_{32}+q_{2}}{a_{22}}\right\rfloor .
$$


Substitute (4.7) into (4.6) and get

$$
s=\left(k_{1}+p_{2} a_{11}+p_{3} a_{31}+p_{4} a_{11}\right) d_{1}+q_{4} d_{2}+q_{3} d_{3},
$$

and $s$ still has its representative node in $\widetilde{\mathbb{L}}$.

2 ) By way of contradiction, assume that there exist two nodes $\left\{k_{1}, k_{2}, k_{3}\right\} \in \widetilde{\mathbb{L}}$ and $\left\{l_{1}, l_{2}, l_{3}\right\} \in \widetilde{\mathbb{L}}$ such that

$$
\begin{aligned}
& k_{1} d_{1}+k_{2} d_{2}+k_{3} d_{3}=l_{1} d_{1}+l_{2} d_{2}+l_{3} d_{3}, \\
& 0 \leq k_{1} \neq l_{1}<\infty, \quad 0 \leq k_{2} \neq l_{2}<a_{22}, \quad 0 \leq k_{3} \neq l_{3}<a_{33} .
\end{aligned}
$$

The case, when one of the differences $k_{j}-l_{j}$ vanishes, will be considered later. Suppose that $k_{1}-l_{1}>0$, and $k_{2}-l_{2}<0, k_{3}-l_{3}<0$. In fact, due to (4.9) we also have to include the upper bound

$$
0<l_{2}-k_{2}<a_{22}, \quad 0<l_{3}-k_{3}<a_{33} .
$$

Rewrite (4.8) as

$$
\left(k_{1}-l_{1}\right) d_{1}=\left(l_{2}-k_{2}\right) d_{2}+\left(l_{3}-k_{3}\right) d_{3},
$$

where $k_{1}-l_{1} \geq a_{11}$, otherwise (due to minimal relations) equation (4.8) would have trivial solution $k_{j}=l_{j}(j=1,2,3)$. But the last contradicts (4.9), namely, $k_{1} \neq l_{1}, k_{2} \neq l_{2}, k_{3} \neq l_{3}$.

If so, represent $k_{1}-l_{1}=u_{1} a_{11}+v_{1}$ with $u_{1} \geq 1,0 \leq v_{1}<a_{11}$, then

$$
\left(u_{1} a_{11}+v_{1}\right) d_{1}=u_{1} a_{22} d_{2}+v_{1} d_{1}=\left(l_{2}-k_{2}\right) d_{2}+\left(l_{3}-k_{3}\right) d_{3} .
$$

Rewrite (4.11) as

$$
\left(l_{3}-k_{3}\right) d_{3}=v_{1} d_{1}+\left(u_{1} a_{22}-\left(l_{2}-k_{2}\right)\right) d_{2},
$$

and note that the both terms on the right-hand side in (4.12) are positive by (4.10),

$$
0<l_{2}-k_{2}<a_{22}<u_{1} a_{22} \text {. }
$$

However, $0<l_{3}-k_{3}<a_{33}$ by (4.10), and (due to minimal relations) equation (4.12) has only a trivial solution, $l_{3}=k_{3}, v_{1}=0, l_{2}=k_{2}+u_{1} a_{22}$. But the last contradicts an inequality (4.13).

Now, consider the case when

$$
a_{33}>k_{3}-l_{3}>0, \quad 0<l_{1}-k_{1}, \quad 0<l_{2}-k_{2}<a_{22},
$$

and write

$$
\left(k_{3}-l_{3}\right) d_{3}=\left(l_{1}-k_{1}\right) d_{1}+\left(l_{2}-k_{2}\right) d_{2} .
$$

But (due to minimal relations) equation (4.14) has only trivial solution $k_{j}=l_{j}$ $(j=1,2,3)$, that contradicts (4.9), namely, $k_{1} \neq l_{1}, k_{2} \neq l_{2}, k_{3} \neq l_{3}$. 
Next, consider the case when

$$
l_{1}-k_{1}=0, \quad 0<l_{2}-k_{2}<a_{22}, \quad a_{33}>k_{3}-l_{3}>0,
$$

and write

$$
\left(k_{3}-l_{3}\right) d_{3}=\left(l_{2}-k_{2}\right) d_{2} .
$$

But (due to minimal relations) equation (4.16) has only a trivial solution, $l_{3}=k_{3}$, $l_{2}=k_{2}$, that contradicts $(4.15)$. For similar reasons the case

$$
k_{3}-l_{3}=0, \quad 0<k_{1}-l_{1}<a_{11}, \quad 0<l_{2}-k_{2}<a_{22},
$$

leads to an equality

$$
\left(k_{1}-l_{1}\right) d_{1}=\left(l_{2}-k_{2}\right) d_{2},
$$

which also has only a trivial solution, $l_{1}=k_{1}, l_{2}=k_{2}$, that contradicts (4.17). Thus, what is left

$$
l_{1}=k_{1}, \quad l_{2}=k_{2}, \quad l_{3}=k_{3},
$$

and the result is proven.

\section{Identities for the Hurwitz zeta function}

As an application, our argument can be deduced to the multiplication theorem in Hurwitz zeta functions. Indeed, combining formulas (3.3) and (3.4), we get an identity

$$
\delta^{n} \sum_{k=1}^{d_{2}-1} \zeta(n, k \delta)=\left(1-\delta^{n}\right) \zeta(n)+\sum_{k=1}^{d_{1}-1} \zeta\left(n, \frac{k}{\delta}\right) .
$$

Another spinoff of formulas (3.3) and (3.4) is a set of identities for Hurwitz zeta functions. For example, consider the numerical semigroup $\langle 3,4\rangle$ with three gaps $\mathbb{N} \backslash\langle 3,4\rangle=\{1,2,5\}$. Substituting it into (3.3) and (3.4), we have

$$
\zeta\left(n, \frac{3}{4}\right)+\zeta\left(n, \frac{6}{4}\right)+\zeta\left(n, \frac{9}{4}\right)=\left(4^{n}-1\right) \zeta(n)-\left(4^{n}+2^{n}+\left(\frac{4}{5}\right)^{n}\right)
$$

and

$$
\zeta\left(n, \frac{4}{3}\right)+\zeta\left(n, \frac{8}{3}\right)=\left(3^{n}-1\right) \zeta(n)-\left(3^{n}+\left(\frac{3}{2}\right)^{n}+\left(\frac{3}{5}\right)^{n}\right),
$$

respectively.

We shall show that the identity (3.3) can be deduced to the multiplication theorem in Hurwitz zeta functions (see, e.g., [1, p.249], [2, (16), p.71]). It is similar for (3.4).

Since $\operatorname{gcd}\left(d_{1}, d_{2}\right)=1$, if $k_{1} d_{1} \equiv k_{2} d_{1}\left(\bmod d_{2}\right)$ then $k_{1} \equiv k_{2}\left(\bmod d_{2}\right)$. Therefore,

$$
\zeta\left(n,\left\{\frac{d_{1}}{d_{2}}\right\}\right)+\zeta\left(n,\left\{\frac{2 d_{1}}{d_{2}}\right\}\right)+\cdots+\zeta\left(n,\left\{\frac{\left(d_{2}-1\right) d_{1}}{d_{2}}\right\}\right)
$$




$$
=\zeta\left(n, \frac{1}{d_{2}}\right)+\zeta\left(n, \frac{2}{d_{2}}\right)+\cdots+\zeta\left(n, \frac{d_{2}-1}{d_{2}}\right),
$$

where $\{x\}$ denotes the fractional part of a real number $x$. There exists a nonnegative integer $a$ such that

$$
\frac{a d_{1}}{d_{2}}<1<\frac{(a+1) d_{1}}{d_{2}} .
$$

Then for any integer $k^{\prime}$ with $a<k^{\prime} \leq d_{2}-1$ there exists a positive integer $l^{\prime}$ such that $1 \leq k^{\prime} d_{1}-l^{\prime} d_{2}<d_{2}$, and

$$
\begin{aligned}
\zeta\left(n, \frac{k^{\prime} d_{1}}{d_{2}}\right)=\zeta\left(n, \frac{k^{\prime} d_{1}-l^{\prime} d_{2}}{d_{2}}\right)-\left(\frac{d_{2}}{k^{\prime} d_{1}-l^{\prime} d_{2}}\right)^{n} \\
\quad-\left(\frac{d_{2}}{k^{\prime} d_{1}-\left(l^{\prime}-1\right) d_{2}}\right)^{n}-\cdots-\left(\frac{d_{2}}{k^{\prime} d_{1}-d_{2}}\right)^{n},
\end{aligned}
$$

where

$$
\frac{k^{\prime} d_{1}-l^{\prime} d_{2}}{d_{2}}=\left\{\frac{k^{\prime} d_{1}}{d_{2}}\right\} .
$$

For any positive integer $r$, there exist integers $x$ and $y$ such that $r=x d_{1}+y d_{2}$. If $0 \leq x<d_{2}$, then $r$ can be expressed uniquely. Thus, if $y \geq 0$, then $r \in S_{2}$. If $y<0$, then $r \notin S_{2}$. The largest integer is given by $\left(d_{2}-1\right) d_{1}-d_{2}$, that is exactly the same as the Frobenius number $F\left(d_{1}, d_{2}\right)$. Thus, $k^{\prime} d_{1}-l^{\prime \prime} d_{2} \notin S_{2}$ for all $l^{\prime \prime}$ with $1 \leq l^{\prime \prime} \leq l^{\prime}$ in (5.1). In addition, if $k_{1} d_{1}-l_{1} d_{2}=k_{2} d_{1}-l_{2} d_{2}$, then by $\operatorname{gcd}\left(d_{1}, d_{2}\right)=1$ we have $d_{1} \mid\left(k_{1}-k_{2}\right)$ and $d_{2} \mid\left(l_{1}-l_{2}\right)$. As $0<k_{1}, k_{2}<d_{2}$ and $0<l_{1}, l_{2}<d_{1}$, we get $k_{1}=k_{2}$ and $l_{1}=l_{2}$. Thus, all such numbers of the form $k d_{1}-l d_{2} \notin S_{2}$ are different.

In $[5,(3.32)]$ for a real $\xi$ and $d=\operatorname{gcd}\left(d_{1}, d_{2}\right)$

$$
\sum_{k=0}^{d_{2}-1}\left\lfloor\frac{k d_{1}+\xi}{d_{2}}\right\rfloor=d\left\lfloor\frac{\xi}{d}\right\rfloor+\frac{\left(d_{1}-1\right)\left(d_{2}-1\right)}{2}+\frac{d-1}{2} .
$$

Hence, by (5.2) with $d=1$ and $\xi=0$, the total number of non-representable positive integers of the form $k d_{1}-l d_{2}\left(a<k<d_{2}, l=1,2, \ldots,\left\lfloor k d_{1} / d_{2}\right\rfloor-1\right)$ is

$$
\sum_{k=1}^{d_{2}-1}\left\lfloor\frac{k d_{1}}{d_{2}}\right\rfloor=\frac{\left(d_{1}-1\right)\left(d_{2}-1\right)}{2},
$$

which is exactly the same as the number of integers without non-negative integer representations by $d_{1}$ and $d_{2}$, that was given by Sylvester in 1882 . Therefore, the right-hand side of $(3.3)$ is

$$
\left(1-\frac{1}{d_{2}^{n}}\right) \zeta(n)-\frac{1}{d_{2}^{n}} \sum_{k_{1}=1}^{d_{2}-1} \zeta\left(n, \frac{k_{1} d_{1}}{d_{2}}\right)
$$




$$
\begin{aligned}
& =\left(1-\frac{1}{d_{2}^{n}}\right) \zeta(n)-\frac{1}{d_{2}^{n}}\left(\sum_{k_{1}=1}^{d_{2}-1} \zeta\left(n,\left\{\frac{k_{1} d_{1}}{d_{2}}\right\}\right)-d_{2}^{n} \sum_{s \in \mathbb{N} \backslash S_{2}} s^{-n}\right) \\
& =\left(1-\frac{1}{d_{2}^{n}}\right) \zeta(n)-\frac{1}{d_{2}^{n}} \sum_{k_{1}=1}^{d_{2}-1} \zeta\left(n, \frac{k}{d_{2}}\right)+\sum_{s \in \mathbb{N} \backslash S_{2}} s^{-n} .
\end{aligned}
$$

On the other hand, the left-hand side of (3.3) is

$$
g_{-n}\left(S_{2}\right)=\sum_{s \in \mathbb{N} \backslash S_{2}} s^{-n} .
$$

Therefore, we obtain that

$$
\sum_{k=1}^{d_{2}} \zeta\left(n, \frac{k}{d_{2}}\right)=d_{2}^{n} \zeta(n),
$$

which is the multiplication theorem in Hurwitz zeta functions.

Acknowledgements. This work was partly done when the first author visited the second author's institute and the second author visited the third author's institute. We would like to thank both researchers for their hospitality and discussion. We thank the anonymous referee for careful reading of our manuscript and many insightful comments and suggestions.

\section{References}

[1] T. M. Apostol: Introduction to analytic number theory. English, Springer, Cham, 1976, DOI: https://doi .org/10.1007/978-1-4757-5579-4.

[2] H. Davenport: Multiplicative number theory. 2nd ed. Rev. by Hugh L. Montgomery. English, vol. 74, Springer, New York, NY, 1980, DOI: https://doi .org/10.1007/978-1-4757-5927-3.

[3] L. G. FeL: Frobenius problem for semigroups $\mathbf{S}\left(d_{1}, d_{2}, d_{3}\right)$, English, Funct. Anal. Other Math. 1.2 (2006), pp. 119-157, ISSN: 1991-0061; 1863-7914/e, DOI: https://doi.org/10.1007/s11853-007-0009-5.

[4] L. G. Fel, B. Y. Rubinstein: Power sums related to semigroups $S\left(d_{1}, d_{2}, d_{3}\right)$. English, Semigroup Forum 74.1 (2007), pp. 93-98, ISSN: 0037-1912; 1432-2137/e, DOI: https://doi.org/10.1007/s00233-006-0658-6.

[5] R. L. Graham, D. E. Knuth, O. Patashnik: Concrete mathematics: a foundation for computer science. 2nd ed. English, 2nd ed., Amsterdam: Addison-Wesley Publishing Group, 1994, pp. xiii + 657, ISBN: 0-201-55802-5/hbk.

[6] Ö. J. Rönseth: A note on Brown and Shiue's paper on a remark related to the Frobenius problem. English, Fibonacci Q. 32.5 (1994), pp. 407-408, ISSN: 0015-0517. 